\title{
Transesophageal echocardiographic strain imaging predicts aortic biomechanics: Beyond diameter
}

Alexander Emmott, MEng, ${ }^{\text {a,b }}$ Haitham Alzahrani, MD, ${ }^{\mathrm{c}}$ Mohammed Alreshidan, MD, ${ }^{\mathrm{c}}$ Judith Therrien, MD, FRCPC, ${ }^{\mathrm{d}}$ Richard L. Leask, PhD, ${ }^{\mathrm{a}, \mathrm{b}}$ and Kevin Lachapelle, MD, FACS, FRCSC

\section{ABSTRACT}

Background: Clinical guidelines recommend resection of ascending aortic aneurysms at diameters $5.5 \mathrm{~cm}$ or greater to prevent rupture or dissection. However, approximately $40 \%$ of all ascending aortic dissections occur below this threshold. We propose new transesophageal echocardiography strain-imaging moduli coupled with blood pressure measurements to predict aortic dysfunction below the surgical threshold.

Methods: A total of 21 patients undergoing aortic resection were recruited to participate in this study. Transesophageal echocardiography imaging of the aortic short-axis and invasive radial blood pressure traces were taken for 3 cardiac cycles. By using EchoPAC (GE Healthcare, Madison, Wis) and postprocessing in MATLAB (MathWorks, Natick, Mass), circumferential stretch profiles were generated and combined with the blood pressure traces. From these data, 2 in vivo stiffness moduli were calculated: the Cardiac Cycle Pressure Modulus and Cardiac Cycle Stress Modulus. From the resected aortic ring, testing squares were isolated for ex vivo mechanical analysis and histopathology. Each square underwent equibiaxial tensile testing to generate stress-stretch profiles for each patient. Two ex vivo indices were calculated from these profiles (energy loss and incremental stiffness) for comparison with the Cardiac Cycle Pressure Modulus and Cardiac Cycle Stress Modulus.

Results: The echo-derived stiffness moduli demonstrate positive significant covariance with ex vivo tensile biomechanical indices: energy loss (vs Cardiac Cycle Pressure Modulus: $\mathrm{R}^{2}=0.5873, P<.0001$; vs Cardiac Cycle Stress Modulus: $\mathrm{R}^{2}=0.6401, P<.0001$ ) and apparent stiffness (vs Cardiac Cycle Pressure Modulus: $\mathrm{R}^{2}=0.2079, P=.0378$; vs Cardiac Cycle Stress Modulus: $\left.\mathrm{R}^{2}=0.3575, P=.0042\right)$. Likewise, these transesophageal echocardiographyderived moduli are highly predictive of the histopathologic composition of collagen and elastin (collagen/elastin ratio vs Cardiac Cycle Pressure Modulus: $\mathrm{R}^{2}=0.6165, P<.0001 ;$ vs Cardiac Cycle Stress Modulus: $\mathrm{R}^{2}=0.6037$, $P<.0001)$.

Conclusions: Transesophageal echocardiography-derived stiffness moduli correlate strongly with aortic wall biomechanics and histopathology, which demonstrates the added benefit of using simple echocardiography-derived biomechanics to stratify patient populations. (J Thorac Cardiovasc Surg 2018;156:503-12)

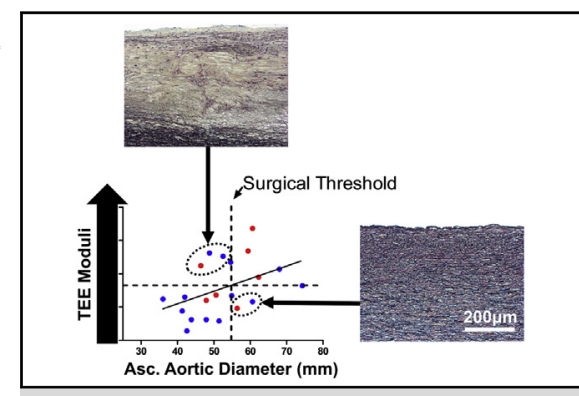

TEE moduli can predict aortic pathology that aortic size cannot.

Central Message

TEE-derived stiffness moduli can identify abnormal aortic mechanical properties that diameter-based guidelines cannot.

\section{Perspective}

Current clinical guidelines assume that there is a critical aortic diameter at which the risk of catastrophic tissue failure increases dramatically. Aortic dilation is a hallmark of the disease but does not represent an intrinsic tissue material property. We have shown that TEE stiffness moduli correlate with ex vivo mechanical indices and can identify patients with pathological wall remodeling.

See Editorial Commentary page 513

\footnotetext{
From the ${ }^{\mathrm{a}}$ Department of Chemical Engineering, and ${ }^{\mathrm{c}}$ Department of Surgery and ${ }^{\mathrm{d}}$ Division of Cardiology, Royal Victoria Hospital, McGill University, Montreal, Quebec, Canada; and ${ }^{\mathrm{b}}$ Research Centre, Montreal Heart Institute, Montreal, Quebec, Canada.

This work is supported by a Natural Sciences and Engineering Research Council of Canada grant. A.E. is supported by a McGill Engineering Doctoral Award.

Received for publication Nov 8, 2017; revisions received Jan 9, 2018; accepted for publication Jan 16, 2018; available ahead of print April 4, 2018.
}

Address for reprints: Kevin Lachapelle, MD, FACS, FRCSC, Cardiac Surgery Department, McGill University Health Centre-Glen Site, 101 Boul. Decarie, C07-1284, H3A 3J1 Montreal, Quebec, Canada (E-mail: kevin.lachapelle@ mcgill.ca). $0022-5223 / \$ 36.00$

Copyright (C) 2018 Published by Elsevier Inc. on behalf of The American Association for Thoracic Surgery

https://doi.org/10.1016/j.jtcvs.2018.01.107 


\section{Abbreviations and Acronyms}

$\mathrm{AA}=$ ascending aortic

$\mathrm{CCPM}=$ Cardiac Cycle Pressure Modulus

CCSM $=$ Cardiac Cycle Stress Modulus

TEE $=$ transesophageal echocardiography

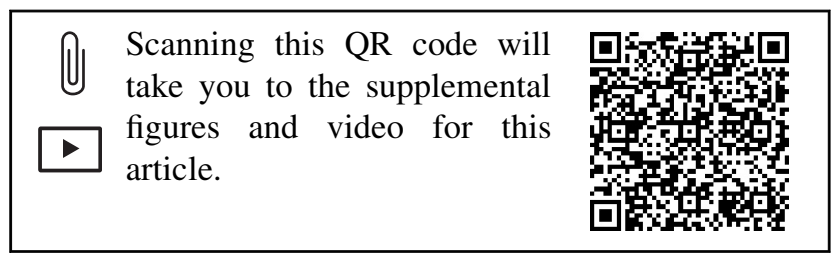

Acute dissection and rupture are usually fatal complications of aortic aneurysms, and prevention is only possible with surgical intervention before these acute complications. Presently, all guidelines use the maximum aortic diameter as the decisional criterion for surgical intervention. ${ }^{1,2}$ Unfortunately, approximately $40 \%$ of patients who present with dissection have aortic diameters below surgical criteria. ${ }^{3}$ Accordingly, novel criteria are needed to identify those who are at risk of dissection or rupture.

Ascending aortic (AA) aneurysms result from pathological remodeling of the vessel wall. ${ }^{4}$ Thinning and fragmentation of elastic lamellae, deposition of collagens, and accumulation of extracellular glycosaminoglycans are the most common characteristics of nonsyndromic and nontraumatic aneurysm formation. ${ }^{5-7}$ This extensive tissue remodeling alters the biomechanical properties of the aorta. ${ }^{8,9}$ Aortic rupture and dissection occur as the result of a loss of mechanical integrity of the vessel wall. Notably, ex vivo mechanical measures of AA tissue, such as stiffness, energy loss, and ultimate strength, have been shown to vary with tissue remodeling. Stiffness ${ }^{10,11}$ and energy $\operatorname{loss}^{12,13}$ both increase with pathological remodeling, whereas strength of the aorta decreases. ${ }^{11}$ These changes in biomechanics were demonstrated using the ex vivo stress-strain relationship based on testing of resected tissue. Reliably estimating these metrics in vivo would provide more information for surgical decisionmaking.

Dynamic echocardiography is routinely used to assess cardiovascular function because of its high temporal resolution without the need to expose patients to contrast agents or ionizing radiation. Dynamic speckle-track strain-imaging of the aorta is an attractive approach to translate the measurement of tissue mechanics from a postoperative ex vivo analysis to a preoperative in vivo assessment.

Previous studies have suggested that aortic stiffness can be deduced from echo strain-imaging. ${ }^{14-18}$ Indeed, transesophageal echocardiography (TEE) studies have revealed that the aortic wall of a subset of patients with bicuspid aortic valve ${ }^{14}$ and patients aged less than 50 years ${ }^{16}$ is less stiff than those with tricuspid aortic valve or aged more than 50 years. However, no direct comparison between intrinsic risk factors, including ex vivo mechanics and underlying wall pathology, has been made.

In this study, we demonstrate that speckle-tracking echocardiography can reliably estimate the biomechanics of aortic tissue obtained ex vivo. Specifically, we used preoperative TEE strain imaging of the aorta with concurrent blood pressure tracing to calculate 2 new stiffness moduli of the aortic wall, which were then compared with ex vivo mechanical analysis and tissue histopathology in patients undergoing aortic resection.

\section{MATERIALS AND METHODS Study Cohort}

In compliance with the Canadian tri-council policy statement on ethical conduct for research involving humans, informed consent was obtained from 21 patients undergoing elective aortic valve or aortic resection surgery. AA diameters ranged from 3.6 to $6.1 \mathrm{~cm}$, that is, from mild to severe dilation.

\section{Ex Vivo Tensile Analysis}

A specimen of the aortic ring was obtained for each patient immediately after resection, clipped for anatomic orientation, and stored in physiologic saline at $4^{\circ} \mathrm{C}$ until further processing and testing. The maximum aortic diameter was measured for each ring before sectioning four $1.5 \times 1.5 \mathrm{~cm}^{2}$ testing squares, equally distributed around the circumference of the aorta representing the 1-anterolateral wall, 2-posterolateral wall, and the 3-inner and 4-outer curvature. Five unique thickness measurements were taken for each testing square using a Mitutoyo Litematic VL-50A constant force digital micrometer (Mitutoyo Corp, Kanagawa, Japan). The testing squares were then connected to an EnduraTEC ELF 3200 planar biaxial tensile tester (Bose, Eden Prairie, Minn) using hooked 4-0 silk sutures in a $37^{\circ} \mathrm{C}$ bath of Ringer's lactate solution. The testing squares were oriented for equibiaxial stretching along their circumferential and longitudinal axes. Each sample was preconditioned for 7 cycles (ie, stretch and relaxation) followed by 3 cycles of data acquisition at a constant displacement rate of $0.1 \mathrm{~mm} / \mathrm{s}$ in the range of $0 \%$ to $60 \%$ strain. The resultant stress-strain relations were analyzed using MATLAB (vR2014a; MathWorks, Natick, Mass). More detailed tensile methodology using this setup has been described previously. ${ }^{10,13,19,20}$

Circumferential ex vivo energy loss and stiffness were calculated from the circumferential engineering stress-strain relation. Energy loss is the percentage of elastic energy needed to stretch the testing square that is dissipated when the tissue is relaxed. The physiologic interpretation of energy loss is the percent loss of elastic recoil energy in the tissue that is not returned to blood flow (ie, maintaining normal Windkessel function). Its physical definition is the ratio of the area between the loading and unloading curve over the area under the loading curve (Figure 1, A). Because aortic tissue has a nonlinear stress-strain curve (Figure 1, $A$ ), stiffness is defined as the slope of a line tangent to the stress-strain loading curve; formally, this parameter is the apparent elastic modulus (defined at $50 \%$ strain) and has been reported previously to describe aortic stiffness in humans. $^{12,13}$

\section{Transesophageal Echocardiographic Strain Imaging}

All TEE imaging was performed at the time of surgery, after administered anesthetic but before the sternotomy, using a GE Vivid 7 
echocardiographic unit (GE Healthcare, Madison, Wis). The TEE probe was inserted into the esophagus to the level of the great vessels to capture the point of maximum aortic dilatation. A 2-dimensional short-axis view of the AA was captured for 3 cardiac cycles, ensuring 1 nontruncated cycle. In tandem, an invasive arterial pressure trace was taken from the patient's radial artery for the same measurement interval.

TEE speckle-tracking strain analysis was performed using GE's EchoPAC software (GE Healthcare). With the use of the Q-Analysis function for the aortic short-axis, markers were manually placed around the aortic circumference and the region of interest (maximal aortic diameter) was adjusted to fit the thickness of the aortic wall. Two-dimensional electrocardiogram-gated radial strain profiles were obtained for 6 segments around the circumference of the aorta (Figure E1). Postprocessing of the radial strain $\left(\varepsilon_{\mathrm{Rad}}=\left[\lambda_{\mathrm{Rad}}-1\right] / 100 \%\right)$ profile was done in MATLAB to generate a global circumferential stretch profile of the aorta for each patient by making the assumptions of (1) tissue incompressibility in the physiologic range $^{21}$ and (2) physiologic aortic stretch is approximately equal in the circumferential and longitudinal axes, ${ }^{22,23}$ and therefore related by the following equation:

$$
\lambda_{\text {Circ }}=\sqrt{\left(1 / \lambda_{\text {Rad }}\right)}
$$

where $\lambda_{\text {Circ }}$ and $\lambda_{\operatorname{Rad}}$ are the circumferential and radial stretches, respectively.

\section{Transesophageal Echocardiography-Derived Stiffness Moduli}

Electrocardiogram gating of both the radial blood pressure and the aortic stretch allowed each profile to be defined on the same cardiac cycle time domain (Figure 1, B). Then, plotting each value of stretch and the phase-shifted pressure at each time step generated a unique pressure-stretch curve for each patient (Figure 1,B). The generated

\section{ex vivo Tensile Mechanics}
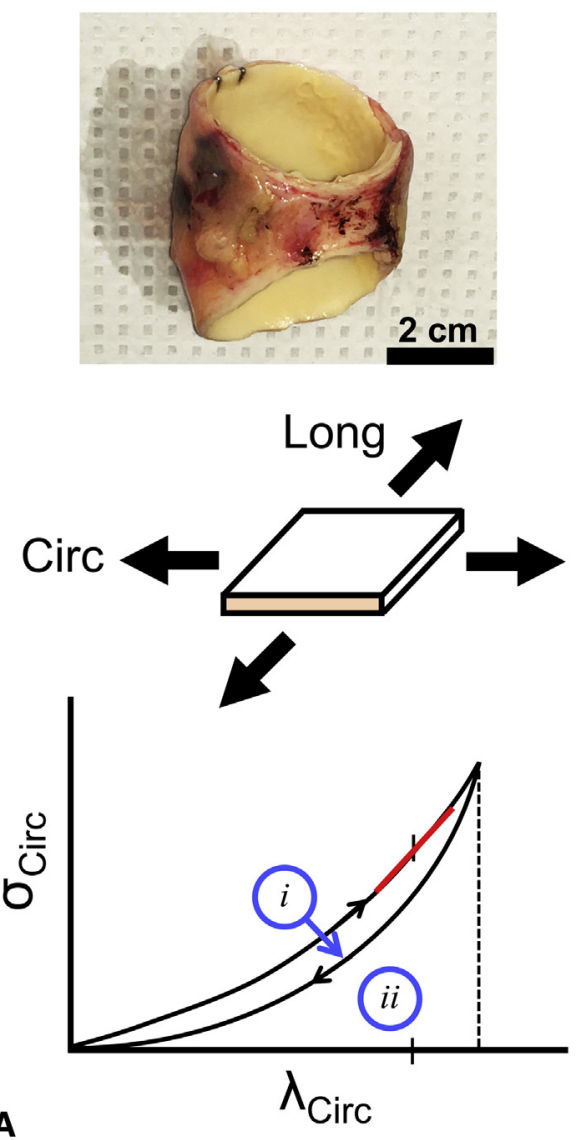

\section{in vivo TEE Mechanics}
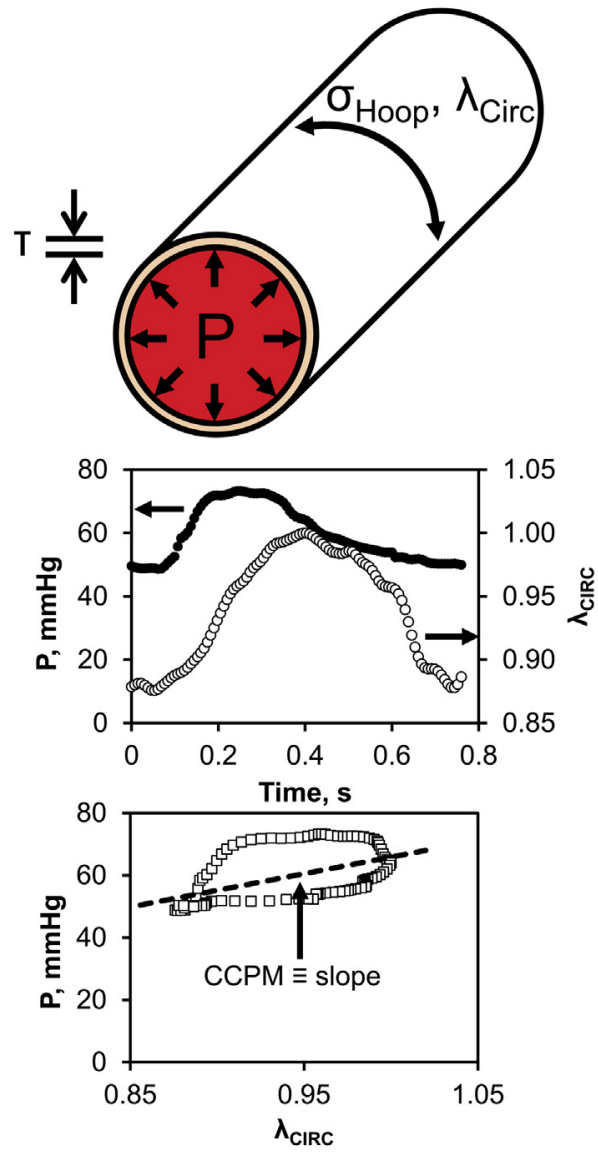

FIGURE 1. AA mechanics using ex vivo tensile analysis and TEE-derived in vivo metrics. A, $1.5 \times 1.5 \mathrm{~cm}^{2}$ testing squares were isolated from an aortic specimen and stretched biaxially along the circumferential (Circ) and longitudinal (Long) axes to obtain the stress-stretch profile of the aortic wall segment. Energy loss is defined as the area between the loading curve and the unloading curve divided by the area under the loading curve: Energy loss = Area(i)/ [Area(i)+Area(ii)] $\times 100 \%$. Stiffness (ie, apparent modulus at 50\% strain) is defined as the slope of a tangent to a defined point on the stress-strain curve. B, In vivo, the aorta expands and recoils with changes in blood pressure leading to increased stresses in the vessel wall. A global (region-averaged) TEE speckle-track strain imaging profile $(\bigcirc)$ and blood pressure profile $(\bullet)$ were plotted for a full cardiac cycle. Blood pressure-stretch curves $(\square)$ were subsequently determined with the slope of the linear fit being defined as a novel TEE modulus, the CCPM. TEE, Transesophageal echocardiography; CCPM, Cardiac Cycle Pressure Modulus. 
TABLE 1. Study cohort demographics—patients are distributed by aortic valve phenotype, sex and age: Preexisting comorbidities and chronic medication use are presented

\begin{tabular}{|c|c|c|c|c|c|c|}
\hline Patient no. & Age, $y$ & Sex & Aortic valve & $\begin{array}{l}\text { Maximum aortic diameter } \\
\qquad(\mathbf{m m})\end{array}$ & Comorbidities & Medication \\
\hline 1 & 44 & $\mathrm{~F}$ & BAV & 36 & AS & BB \\
\hline 2 & 50 & $\mathrm{~F}$ & BAV & 42 & AS & \\
\hline 3 & 52 & $\mathrm{~F}$ & BAV & 42 & AS, HT & ACEI, BB \\
\hline 4 & 56 & $\mathrm{~F}$ & BAV & 42 & HT, AS & $\mathrm{BB}$ \\
\hline 5 & 81 & $\mathrm{~F}$ & BAV & 68 & AS & \\
\hline 6 & 22 & M & BAV & 75 & $\mathrm{FH}$, latent tuberculosis & BB \\
\hline 7 & 38 & M & BAV & 58 & AI & $\mathrm{ARB}$, statin \\
\hline 8 & 46 & M & BAV & 36 & AS, MI & ACEI, BB, CCB, statin \\
\hline 9 & 57 & M & MECH (BAV) & 55 & AVR at $22 \mathrm{y}$ & ACEI \\
\hline 10 & 61 & M & BAV & 45 & AI, hypothyroidism & Levothyroxine \\
\hline 11 & 69 & M & BAV & 47 & AS & Statin \\
\hline 12 & 72 & M & BAV & 49 & AS, MI & \\
\hline 13 & 76 & M & BAV & 57 & AS, AFib, asthma & $\mathrm{ARB}, \mathrm{CCB}$, digoxin \\
\hline 14 & 79 & M & BAV & 53 & AS, HTN, DM & ACEI, amiodarone \\
\hline 15 & 71 & $\mathrm{~F}$ & TAV & 61 & AI, COPD, MI & $\mathrm{BB}$ \\
\hline 16 & 86 & $\mathrm{~F}$ & TAV & 60 & AI, HT & ACEI, BB \\
\hline 17 & 88 & $\mathrm{~F}$ & TAV & 55 & AI & ACI, CCB \\
\hline 18 & 57 & M & TAV & 40 & AI, OI & $\mathrm{BB}$ \\
\hline 19 & 58 & M & TAV & 53 & AI & ARB \\
\hline 20 & 66 & M & TAV & 50 & AS, HTN & $\mathrm{ARB}, \mathrm{CCB}$ \\
\hline 21 & 77 & M & TAV & 50 & AI, DM, HT & ACEI, CCB, statin \\
\hline
\end{tabular}

$F$, Female; $B A V$, bicuspid aortic valve; $A S$, aortic stenosis; $B B$, beta-blocker; $H T$, hypertension; $A C E I$, angiotensin-converting enzyme inhibitor; $M$, male; $F H$, family history; $A I$, aortic insufficiency; $A R B$, angiotensin receptor blocker; $M I$, myocardial infarction; $C C B$, calcium channel blocker; $M E C H$, mechanical valve; $A V R$, aortic valve replacement; $A F i b$, atrial fibrillation; $H T N$, hypertension; $D M$, diabetes mellitus; $T A V$, tricuspid aortic valve; $C O P D$, chronic obstructive pulmonary disease; $O I$, osteogenesis imperfecta

curve creates a hysteresis loop similar to the ex vivo stress-strain curve. However, the shapes of the curves are distinctly different because of the assumption of translating blood pressure to a tissue stress and the limitations in the scale and rate of strain produced in vivo. Therefore, we use the average slope of the pressure stretch-loop as a metric for comparison. This novel mechanical parameter, termed the "Cardiac Cycle Pressure Modulus" (CCPM), is in effect a simple measure of the average aortic stiffness that does not account for variations in tissue thickness between patients. This measure captures the dynamics of both the loading (increasing tissue stresses) and unloading (decreasing tissue stresses) states of the cycle but is notably different from the ex vivo analysis where, by convention, stiffness is evaluated only on the loading curve.

The circumferential stress in the aortic wall was then estimated by applying Laplace's Law, which states that for thin-walled, isotropic, cylindrical vessels, the circumferential wall stress (formally "Hoop Stress", $\left.\sigma_{\text {Hoop }}\right)$ is related to the luminal blood pressure (P) by the following:

$$
\sigma_{\text {Hоop }}=\mathrm{PD} /(2 \tau)
$$

where $\mathrm{D}$ is the aortic diameter (ie, maximum echo-measured aortic diameter) and $\tau$ is the aortic wall thickness. By using this relation and converting the pressure-stretch profile to a hoop stress-stretch profile, we generated a second stiffness modulus, termed the "Cardiac Cycle Stress Modulus" (CCSM) that has the following definition:

$$
\mathrm{CCSM} \equiv \mathrm{CCPM} \times \mathrm{D} /(2 \tau)
$$

This measure requires a priori knowledge of the aortic thickness that, for this study, was the ex vivo tissue thickness. This methodology is further demonstrated in Video 1.

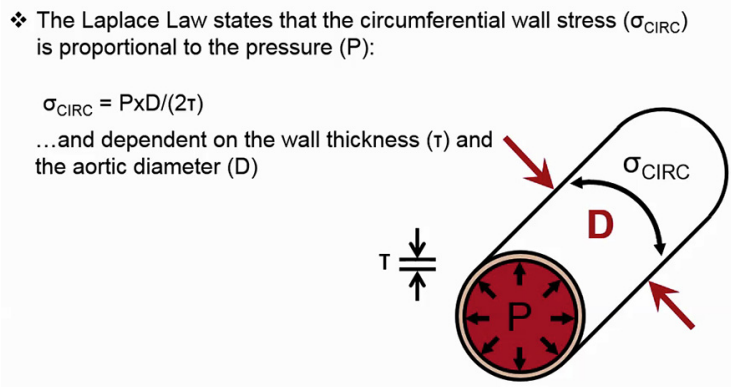

Our second modulus, the Cardiac Cycle Stress Modulus (CCSM), uses this definition:

\section{$\mathrm{CCSM} \equiv \mathrm{CCPM} \times \mathrm{D} /(2 \mathrm{~T})$}

VIDEO 1. Echocardiographic strain and blood pressure data acquisition and procedural calculation of novel in vivo stiffness moduli. Video available at: http://www.jtcvsonline.org/article/S0022-5223(18)30722-0/fulltext. 


\section{Energy Loss}

\section{Stiffness}
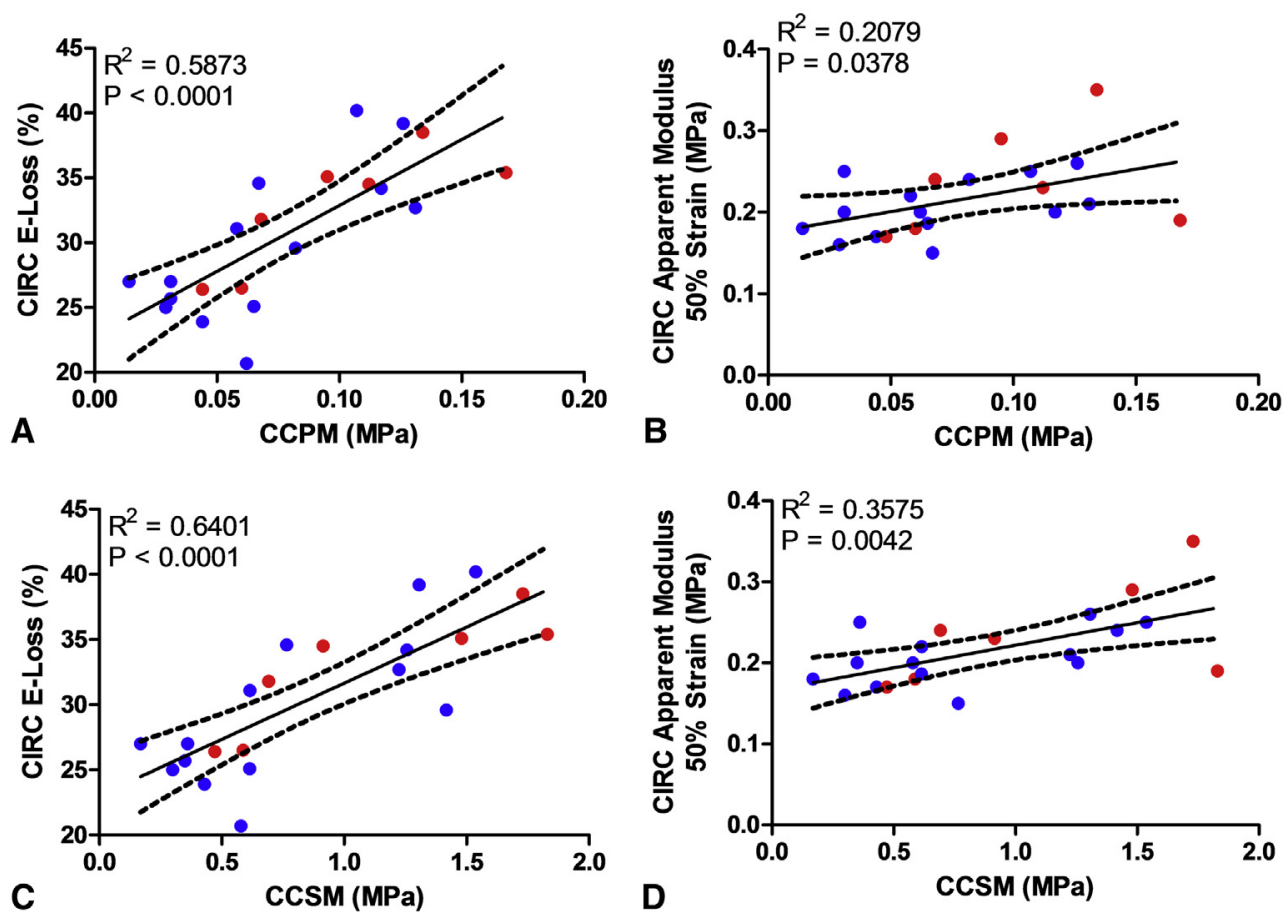

FIGURE 2. Comparison of TEE-derived moduli of with ex vivo measures of energy loss and stiffness by regression analysis. A, Ex vivo circumferential energy loss and (B) circumferential stiffness versus CCPM. C, Ex vivo circumferential energy loss and (D) circumferential stiffness versus CCSM. Data points are distinguished by aortic valve phenotype: tricuspid aortic valve (red dot) and bicuspid aortic valve (blue dot). Circ, Circumferential; CCPM, Cardiac Cycle Pressure Modulus; CCSM, Cardiac Cycle Stress Modulus.

\section{Histology}

A small piece of aortic tissue adjacent to each testing square was preserved in $10 \%(\mathrm{v} / \mathrm{v})$ buffered formalin. The tissue was paraffin embedded, and $7-\mu \mathrm{m}$ sections were prepared and stained with Movat's pentachrome. Two $10 \times$-magnified images were obtained for each section: one representing the intima-media and the other representing the media-adventitia. Percent micrograph field coverage of medial elastin and collagens was quantified for each image using ImageJ. A single data point per patient represents an average histologic quantification of all regions of inquiry around the aortic circumference.

\section{Statistical Analysis}

Statistical analysis was performed using GraphPad Prism 5.01 (GraphPad Software Inc, San Diego, Calif). Linear regression analysis was used to determine covariance. Covariance plots are presented with a linear regression line (solid, black) and a 95\% confidence interval (dotted, black).

\section{RESULTS}

A total of 21 patients were recruited for this study. Table 1 summarizes our study group by aortic valve phenotype, age, sex, maximum aortic diameter, preexisting comorbidities, and chronic medication. A total of 7 patients with tricuspid aortic valves and 14 patients with bicuspid aortic valves were included with mean ages of $72 \pm 12$ years and $57 \pm 17$ years, respectively. For 6 patients, fewer than 4 quadrants were available for ex vivo testing because of specimen size.
Transesophageal Echocardiography Strain ImagingDerived Measures of Impaired Aortic Biomechanics

The CCPM (Figure 2,A) and CCSM (Figure 2, C) demonstrated a strongly significant covariance with ex vivo circumferential energy loss $\left(\mathrm{R}^{2}=0.5873\right.$, $P<.0001)$ and $\left(\mathrm{R}^{2}=0.6401, P<.0001\right)$, respectively, with larger moduli being measured in aortas with increased energy loss.

The 2 TEE-derived moduli were compared with ex vivo aortic wall stiffness, with ex vivo stiffness being defined as the apparent modulus at $50 \%$ strain. Our findings demonstrate that CCPM demonstrates a positive, significant covariance with the ex vivo apparent elastic modulus $\left(\mathrm{R}^{2}=0.2079, P=.0378\right.$ ) (Figure $\left.2, B\right)$. Likewise, we observed a significant positive covariance when comparing CCSM with the ex vivo apparent modulus $\left(\mathrm{R}^{2}=0.3575\right.$, $P=.0042$ ) (Figure 2, $D$ ).

\section{Transesophageal Echocardiography Strain Imaging-Derived Measures as a Predictor of Aortic Histopathology}

The collagen/elastin ratio, which is a unitless fraction of the 2 species, co-varied significantly with the CCPM (Figure 3, A) and CCSM (Figure 3,B) $\left(\mathrm{R}^{2}=0.6165\right.$, $P<.0001)\left(\mathrm{R}^{2}=0.6037, P<.0001\right)$, respectively, revealing 

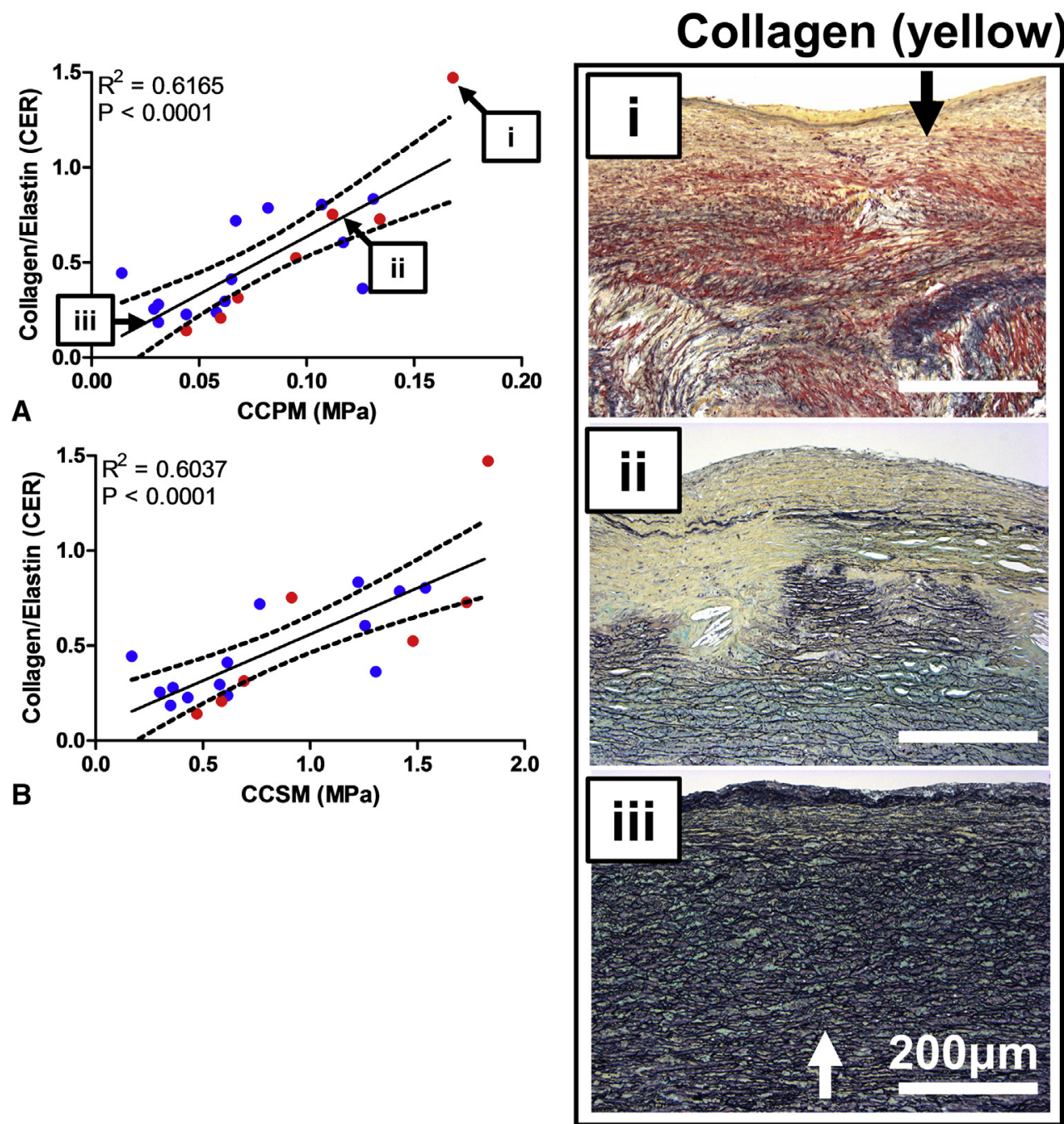

\section{Elastin (black)}

FIGURE 3. Comparison of TEE-derived moduli with aortic wall histopathology by regression analysis. A, Aortic wall collagen/elastin versus CCPM. B, Aortic wall collagen/elastin versus CCSM. Representative Movat-stained histologic images of 3 patients reveal varied levels of collagen deposition (stained yellow) and intact or disrupted elastic sheets (stained black). Data points are distinguished by aortic valve phenotype: tricuspid aortic valve (red dot) and bicuspid aortic valve (blue dot). CCPM, Cardiac Cycle Pressure Modulus; CCSM, Cardiac Cycle Stress Modulus.

that patients with higher proportions of medial collagens relative to elastin had greater TEE-measured moduli. Representative histologic images are presented in Figure 3 to highlight the range of tissue remodeling observed in this patient cohort. This range includes littleto-no loss of the de novo aortic wall composition to severe medial degeneration with nearly complete loss of the elastic structure with sizeable deposition of collagens.

\section{Aortic Diameter as a Predictor of Impaired Aortic Biomechanics}

In vivo aortic diameter was significantly correlated with ex vivo circumferential energy loss $\left(\mathrm{R}^{2}=0.3800\right.$, $P=.0029$ ) (Figure $4, A)$, but not with ex vivo measured circumferential stiffness $\left(\mathrm{R}^{2}=0.1486, P=.0855\right)$ (Figure 4, B). Aortic diameter was modestly correlated with aortic wall histopathology, with larger diameter aortas containing a greater proportion of collagen/elastin $\left(\mathrm{R}^{2}=0.2155, P=.0340\right.$ ) (Figure $\left.4, C\right)$.

Finally, to illustrate how the CCPM and CCSM can be applied to improve patient outcome, these values were plotted as a function of aortic diameter to establish a 2-dimensional risk assessment (Figure 5, $A$ and $B$, respectively). The CCPM or CCSM as the vertical axis describes the risk associated with impaired mechanical function and pathological remodeling that can occur irrespective of aortic diameter. The correlations are low. This indicates that both indices identify features that 

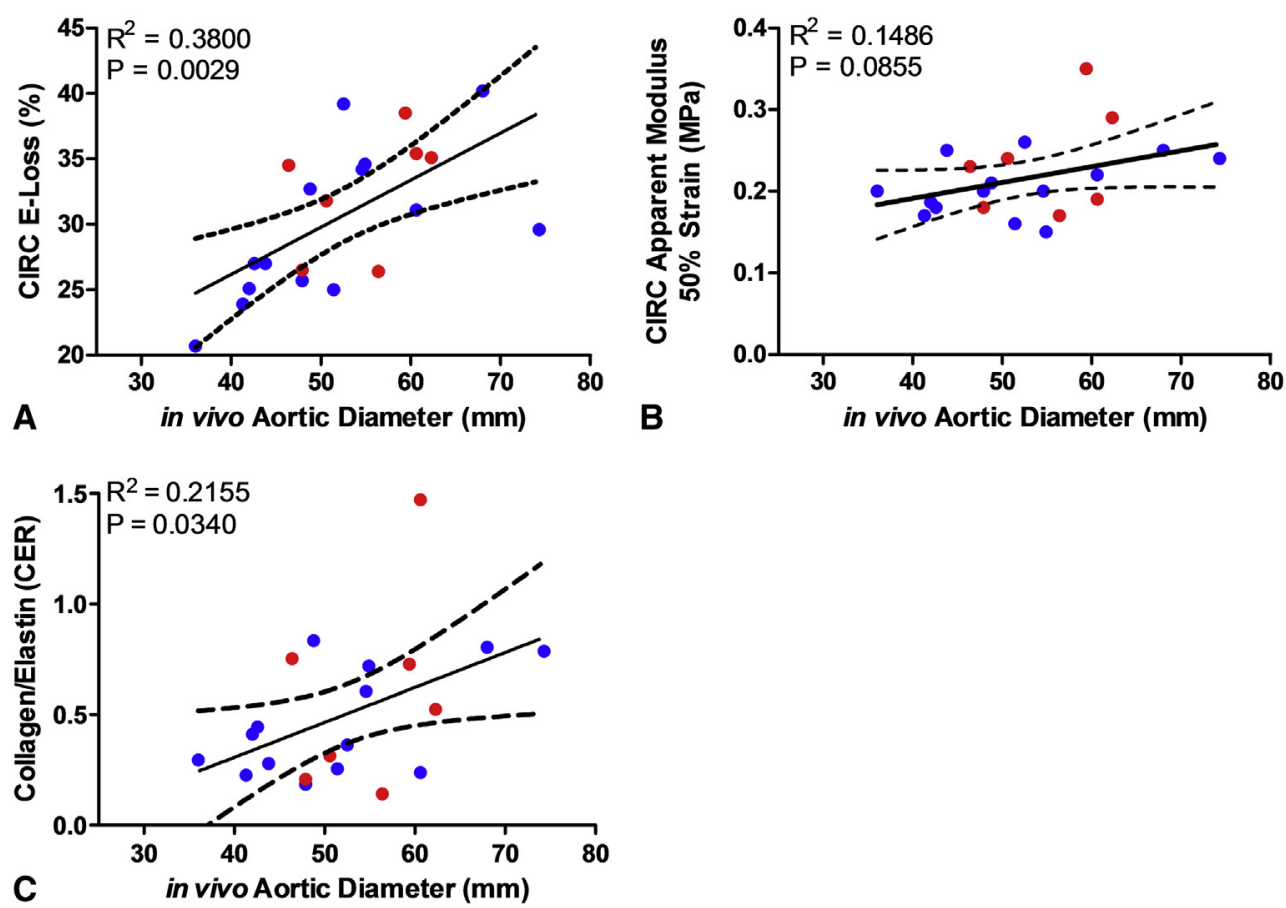

FIGURE 4. Comparison of aortic size with ex vivo biomechanics and histopathology. A, Ex vivo circumferential energy loss versus in vivo aortic diameter. B, Ex vivo circumferential stiffness versus in vivo aortic diameter. C, Aortic wall collagen/elastin versus in vivo aortic diameter. Data points are distinguished by aortic valve phenotype: tricuspid aortic valve (red dot) and bicuspid aortic valve (blue dot). Circ, Circumferential.

are not identical with aortic diameter and therefore, potentially, could contribute independent information as to risk. Indeed, 3 patients below the surgical threshold with large echo moduli were identified. Histologic assessment of these patients confirmed a pathological disruption of the elastic structure with accumulated collagen deposition (Figure $5, *, \dagger$, and $\ddagger$ ). Furthermore, 2 patients above the surgical threshold with low echo moduli were identified as having a less pathological aortic wall with intact elastic lamellae and little collagen (Figure 5, $\S$ and $\|$ ).

A summary of the comparisons between the TEE-derived aortic measurements and moduli and ex vivo measures, including the tensile mechanics and wall histopathology, is presented in Table 2.

\section{DISCUSSION}

Current clinical guidelines assume that there is a critical aortic diameter at which the risk of catastrophic tissue failure increases dramatically. ${ }^{24,25}$ Aortic dilation is, unquestionably, a hallmark of the disease but does not represent an intrinsic tissue material property. Aortic dilatation also fails to relay the magnitude of stress or concentration of stress in the aortic wall. Accordingly, our aim was to use TEE speckle tracking with blood pressure to devise novel in vivo stiffness moduli based on tissue biomechanics, which might help stratify at-risk patients beyond aortic diameter alone.
By using 2-dimensional short-axis AA strain imaging with concurrent blood pressure tracing, we developed moduli of the pressure-stretch (CCPM) and stress-stretch (CCSM) cardiac cycle average loop. Both the CCPM and CCSM significantly predicted the variance of ex vivo energy loss. Likewise, the CCPM and CCSM significantly predict ex vivo stiffness. Both indices performed better than aortic diameter.

Both echo moduli and aortic diameter were more closely related to energy loss than to ex vivo stiffness. These observations are of interest because other studies have shown that energy loss is representative of aortic wall histopathol$\operatorname{ogy}^{12,13,26}$ and has been found to increase in regions of chronic aortic dissection, ${ }^{27}$ whereas aortic stiffness did not significantly correlate with tissue histopathology. ${ }^{13}$ Moreover, ex vivo stiffness (50\% apparent modulus) is defined at a single strain value. Accordingly, small variations in ex vivo measurements, such as the suture placement during tensile testing, can have a considerable impact on the calculated stiffness because if its highly nonlinear relationship with strain. By contrast, ex vivo energy loss is less susceptible to these pitfalls because it is, by definition, a self-normalizing value, averaged over a full stress-stretch cycle.

CCSM was more highly correlated with ex vivo aortic energy loss and stiffness than the CCPM. This may be due to including actual aortic anatomic dimension in the calculation of CCSM rather than simple blood pressure in 


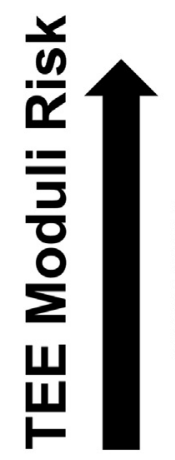

A
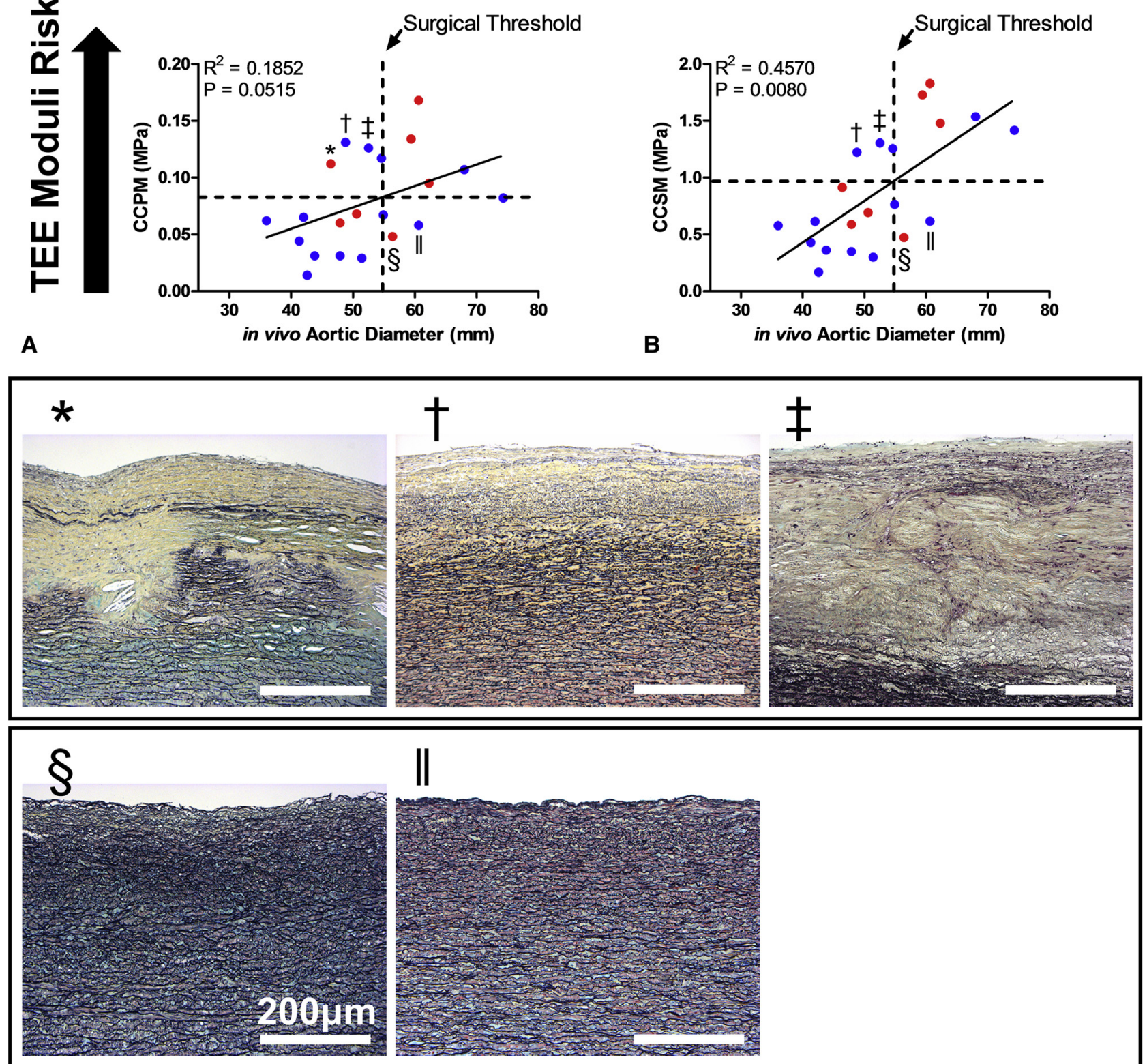

FIGURE 5. Comparison of TEE-derived moduli with aortic size as a method to identify at-risk patients. A, CCPM versus in vivo aortic diameter. B, CCSM versus in vivo aortic diameter. The vertical dotted line is the current surgical threshold of $5.5 \mathrm{~cm}$ or greater, and the horizontal dotted line is defined at the intercept of the linear regression and the surgical threshold. Histopathology panels of Movat-stained sections of the AA wall: high echo moduli, low AA diameter (patients: *,,,$\ddagger$ ); low echo moduli, large AA diameter (patients: $\S, \|$ ). Data points are distinguished by aortic valve phenotype: tricuspid aortic valve $($ red dot) and bicuspid aortic valve (blue dot). TEE, Transesophageal echocardiography; CCPM, Cardiac Cycle Pressure Modulus.

calculation of CCPM. On the other hand, CCPM has the practical advantage that it requires only standard blood pressure tracing and TEE strain imaging whereas CCSM requires measurement of the aortic wall thickness that, for this study, was taken ex vivo. For the CCSM to become a fully in vivo measure, an accurate methodology to measure aortic tissue thickness must be established. Magnetic resonance imaging has sufficient resolution, ${ }^{28}$ whereas axial highfrequency echo resolution is approximately $0.25 \mathrm{~mm}^{29}$ and therefore cannot precisely measure regional and interpatient differences in aortic thickness..$^{10,30}$

Histopathologic changes in the AA wall are a central marker of the disease process in degenerative AA aneurysm. ${ }^{4}$ In particular, the content of the structural proteins, collagen, and elastin is altered significantly in the pathological remodeling process of the AA wall. ${ }^{4}$ Both the CCSM and CCPM correlated strongly with the collagen/ elastin ratio in the AA. Aortic diameter also significantly 
TABLE 2. Aortic size, mechanical and histopathologic comparisons, and corresponding regression coefficients and $P$ values

\begin{tabular}{|c|c|c|c|}
\hline & \multicolumn{2}{|c|}{ Ex vivo tensile mechanics } & \multirow{3}{*}{$\begin{array}{l}\text { Histopathology } \\
\text { Collagen/elastin }\end{array}$} \\
\hline & \multirow[b]{2}{*}{ Energy loss } & \multirow{2}{*}{$\begin{array}{c}\text { Stiffness } \\
\text { (Apparent modulus) }\end{array}$} & \\
\hline & & & \\
\hline In vivo & $* *$ & NS & $*$ \\
\hline Aortic diameter & $\mathrm{R}^{2}=0.3800, P=.0029$ & $\mathrm{R}^{2}=0.1486, P=.0855$ & $\mathrm{R}^{2}=0.2155, P=.0340$ \\
\hline \multicolumn{4}{|c|}{ In vivo TEE mechanics } \\
\hline \multirow[t]{2}{*}{ СCРM } & $* * *$ & $*$ & $* * *$ \\
\hline & $\mathrm{R}^{2}=0.5873, P<.0001$ & $\mathrm{R}^{2}=0.2079, P=.0378$ & $\mathrm{R}^{2}=0.6165, P<.0001$ \\
\hline \multirow[t]{2}{*}{ CCSM } & $* * *$ & $* *$ & $* * *$ \\
\hline & $\mathrm{R}^{2}=0.6401, P<.0001$ & $\mathrm{R}^{2}=0.3575, P=.0042$ & $\mathrm{R}^{2}=0.6037, P<.0001$ \\
\hline
\end{tabular}

correlated with collagen/elastin, but the strength of the correlation was less.

Other TEE measures have been used to describe stiffness in the ascending aorta. ${ }^{14-18}$ The most common is the $\beta$ stiffness index $\left(\beta=\ln \left[\mathrm{P}_{\mathrm{S}} / \mathrm{P}_{\mathrm{D}}\right] /\left[\left(\mathrm{D}_{\max }-\mathrm{D}_{\min }\right) / \mathrm{D}_{\min }\right]\right)$, a 2-point measure that relates the systolic/diastolic pressure ratio to the change in AA diameter (D). ${ }^{31}$ Unlike the moduli presented in this study, $\beta$ does not account for the temporal component of strain or pressure. In a study of 17 patients, Alreshidan and colleagues ${ }^{18}$ have shown that the $\beta$ stiffness index is not predictive of regional ex vivo stiffness in the AA. In this cohort, we observed that $\beta$ did not significantly correlate with ex vivo stiffness and only moderately correlated with energy loss and histopathology, performing worse than both the CCPM and the CCSM (Figure E2).

Aortic diameter is, unquestionably, an important maker of the risk of rupture or dissection. However, equally clearly, it is not sufficient because at least $40 \%$ of these catastrophic events occur at an aortic diameter below the threshold for surgical intervention. To be of clinical value, any additional marker must contribute new information as to risk. That is why the relatively low correlation between CCSM and CCPM with aortic diameter is encouraging because it suggests that if CCSM and CCPM are shown in prospective studies to be significant markers of risk that they may well contribute prognostic information that is independent of, and therefore additive to, aortic diameter.

\section{Study Limitations}

Our cohort is similar in size to others that have been designed to compare in vivo parameters with ex vivo measures of risk..$^{10,11,18}$ It was designed to validate 2 novel noninvasive measures against ex vivo measures of aortic wall function and structure. It was not designed to determine whether these novel measures contribute significant prognostic information as to the risk of a catastrophic aortic event in patients with aneurysms of the ascending aorta. Second, assigning 1 modulus value per patient relies on the average strain profile around the circumference of the aorta, which was rarely uniform regionally. This potentially limits our understanding of the regional differences in AA wall behavior and its connection to patient risk. Finally, this method relies on a nontruncated short-axis view of the aorta. Because of a known blind spot at the level of the tracheal carina, the distal ascending aorta and aortic arch are obscured from TEE view.

Although energy loss has been reported as a robust mechanical measure that corresponds to the pathological state of the aortic wall, no prospective study has been conducted that links energy loss with clinical occurrence of acute dissection or rupture. Furthermore, the limited range of strain during the cardiac cycle makes estimating in vivo energy loss difficult.

\section{CONCLUSIONS}

This study involved a cohort of patients with a broad range of AA dilation above and below surgical criteria of $5.5 \mathrm{~cm}$ or greater. We have demonstrated that 2 novel TEE-derived strain imaging moduli can be obtained noninvasively and correlate highly with ex vivo mechanics and histopathology. AA diameter, which is used as surgical criteria, is considerably less predictive of these properties. The present findings support prospective testing of CCPM and CCSM as novel noninvasive markers of the risk of aortic rupture or dissection.

\section{Conflict of Interest Statement}

Authors have nothing to disclose with regard to commercial support.

\section{References}

1. Hiratzka LF, Bakris GL, Beckman JA, Bersin RM, Carr VF, Casey DE, et al. 2010 ACCF/AHA/AATS/ACR/ASA/SCA/SCAI/SIR/STS/SVM guidelines for the diagnosis and management of patients with thoracic aortic disease: a report of the American College of Cardiology Foundation/American Heart Association task force on practice guidelines, American Association for Thoracic Surgery, American College of Radiology, American Stroke Association, Society of Cardiovascular Anesthesiologists, Society for Cardiovascular Angiography and Interventions, Society of Interventional Radiology, Society of Thoracic Surgeons, and Society for Vascular Medicine. Circulation. 2010;121:e266-369. 
2. Boodhwani M, Andelfinger G, Leipsic J, Lindsay T, McMurtry MS, Therrien J, et al. Canadian Cardiovascular Society position statement on the management of thoracic aortic disease. Can J Cardiol. 2014;30:577-89.

3. Pape LA, Tsai TT, Isselbacher EM, Oh JK, O'Gara PT, Evangelista A, et al Aortic diameter $>$ or $=5.5 \mathrm{~cm}$ is not a good predictor of type A aortic dissection: observations from the international registry of acute aortic dissection (IRAD). Circulation. 2007;116:1120-7.

4. Emmott A, Garcia J, Chung J, Lachapelle K, El-Hamamsy I, Mongrain R, et al Biomechanics of the ascending thoracic aorta: a clinical perspective on engineering data. Can J Cardiol. 2016;32:35-47.

5. Carlson RG, Lillehei CW, Edwards JE. Cystic medial necrosis of the ascending aorta in relation to age and hypertension. Am J Cardiol. 1970;25:411-5.

6. Hirst AE Jr, Johns VJ Jr, Kime SW Jr. Dissecting aneurysm of the aorta: a review of 505 cases. Medicine. 1958;37:217-79.

7. Humphrey JD. Possible mechanical roles of glycosaminoglycans in thoracic aortic dissection and associations with dysregulated transforming growth factor-beta. J Vasc Res. 2013;50:1-10.

8. Weisbecker H, Viertler C, Pierce DM, Holzapfel GA. The role of elastin and collagen in the softening behavior of the human thoracic aortic media. J Biomech. 2013;46:1859-65.

9. Gasser TC, Ogden RW, Holzapfel GA. Hyperelastic modelling of arterial layers with distributed collagen fibre orientations. J R Soc Interface. 2006;3:15-35.

10. Choudhury N, Bouchot O, Rouleau L, Tremblay D, Cartier R, Butany J, et al. Local mechanical and structural properties of healthy and diseased human ascending aorta tissue. Cardiovasc Pathol. 2009;18:83-91.

11. Vorp DA, Schiro BJ, Ehrlich MP, Juvonen TS, Ergin MA, Griffith BP. Effect of aneurysm on the tensile strength and biomechanical behavior of the ascending thoracic aorta. Ann Thorac Surg. 2003:75:1210-4.

12. Chung J, Lachapelle K, Cartier R, Mongrain R, Leask RL. Loss of mechanical directional dependency of the ascending aorta with severe medial degeneration. Cardiovasc Pathol. 2017;26:45-50.

13. Chung J, Lachapelle K, Wener E, Cartier R, De Varennes B, Fraser R, et al. Energy loss, a novel biomechanical parameter, correlates with aortic aneurysm size and histopathologic findings. J Thorac Cardiovasc Surg. 2014;148:1082-9.

14. Petrini J, Jenner J, Rickenlund A, Eriksson P, Franco-Cereceda A, Caidahl K, et al. Elastic properties of the descending aorta in patients with a bicuspid or tricuspid aortic valve and aortic valvular disease. J Am Soc Echocardiogr 2014;27:393-404.

15. Teixeira R, Moreira N, Baptista R, Barbosa A, Martins R, Castro G, et al. Circumferential ascending aortic strain and aortic stenosis. Eur Heart $J$ Cardiovasc Imaging. 2013;14:631-41.

16. Oishi Y, Miyoshi H, Mizuguchi Y, Iuchi A, Nagase N, Oki T. Aortic stiffness is strikingly increased with age $>/=50$ years in clinically normal individuals and preclinical patients with cardiovascular risk factors: assessment by the new technique of 2D strain echocardiography. J Cardiol. 2011;57:354-9.

17. Bieseviciene M, Vaskelyte JJ, Mizariene V, Karaliute R, Lesauskaite V, Verseckaite R. Two-dimensional speckle-tracking echocardiography for evaluation of dilative ascending aorta biomechanics. BMC Cardiovasc Disord. 2017:17:27.
18. Alreshidan M, Shahmansouri N, Chung J, Lash V, Emmott A, Leask RL, et al Obtaining the biomechanical behavior of ascending aortic aneurysm via the use of novel speckle tracking echocardiography. J Thorac Cardiovasc Surg 2017; $153: 781-8$.

19. Tremblay D, Cartier R, Mongrain R, Leask RL. Regional dependency of the vascular smooth muscle cell contribution to the mechanical properties of the pig ascending aortic tissue. J Biomech. 2010;43:2448-51.

20. Tremblay D, Leask RL. Remodelling and pathology development associated with aneurysmal ascending aortic tissues. Can J Chem Eng. 2011;89:13-22.

21. Carew TE, Vaishnav RN, Patel DJ. Compressibility of the arterial wall. Circ Res 1968;23:61-8.

22. Bell V, Mitchell WA, Sigurethsson S, Westenberg JJ, Gotal JD, Torjesen AA, et al. Longitudinal and circumferential strain of the proximal aorta. J Am Heart Assoc. 2014;3:e01536.

23. Wittek A, Karatolios K, Fritzen CP, Bereiter-Hahn J, Schieffer B, Moosdorf R, et al. Cyclic three-dimensional wall motion of the human ascending and abdom inal aorta characterized by time-resolved three-dimensional ultrasound speckle tracking. Biomech Model Mechanobiol. 2016;15:1375-88.

24. Coady MA, Rizzo JA, Hammond GL, Mandapati D, Darr U, Kopf GS, et al. What is the appropriate size criterion for resection of thoracic aortic aneurysms? J Thorac Cardiovasc Surg. 1997;113:476-91.

25. Elefteriades JA. Natural history of thoracic aortic aneurysms: indications for surgery, and surgical versus nonsurgical risks. Ann Thorac Surg. 2002;74:S1877-80; discussion S1892-8.

26. Shahmansouri N, Alreshidan M, Emmott A, Lachapelle K, Cartier R, Leask RL, et al. Evaluating ascending aortic aneurysm tissue toughness: dependence on collagen and elastin contents. J Mech Behav Biomed Mater. 2016;64: $262-71$

27. Emmott A, El-Hamamsy I, Leask RL. Histopathological and biomechanical properties of the aortic wall in 2 patients with chronic type A aortic dissection. Cardiovasc Pathol. 2017;29:48-52

28. Li AE, Kamel I, Rando F, Anderson M, Kumbasar B, Lima JA, et al Using MRI to assess aortic wall thickness in the multiethnic study of atherosclerosis: distribution by race, sex, and age. AJR Am J Roentgenol. 2004;182 $593-7$

29. Gradus-Pizlo I, Bigelow B, Mahomed Y, Sawada SG, Rieger K, Feigenbaum H. Left anterior descending coronary artery wall thickness measured by high frequency transthoracic and epicardial echocardiography includes adventitia Am J Cardiol. 2003;91:27-32.

30. Pham T, Martin C, Elefteriades J, Sun W. Biomechanical characterization of ascending aortic aneurysm with concomitant bicuspid aortic valve and bovine aortic arch. Acta Biomater. 2013;9:7927-36.

31. O'Rourke MF, Staessen JA, Vlachopoulos C, Duprez D, Plante GE. Clinical applications of arterial stiffness; definitions and reference values. Am J Hypertens 2002;15:426-44.

Key Words: ascending aorta, aneurysm, biomechanics, echocardiography, histology 


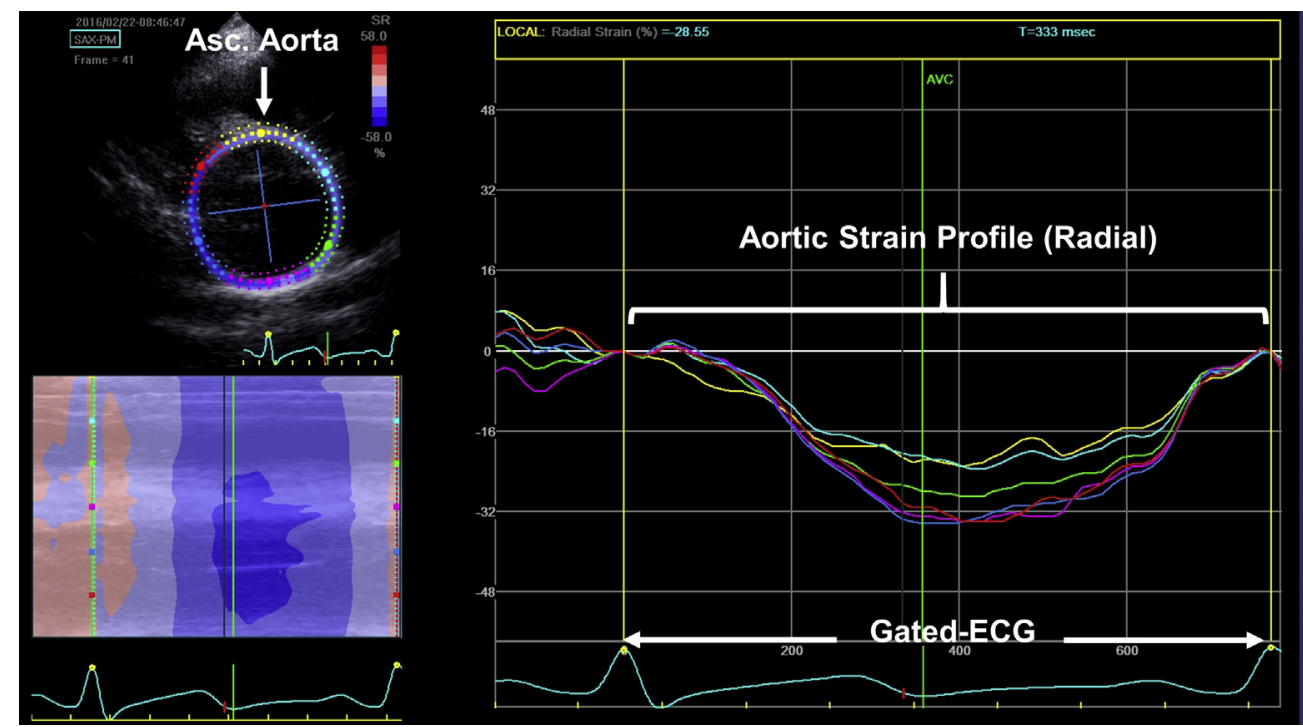

FIGURE E1. Representative electrocardiogram-gated radial strain profile of the ascending aorta using EchoPAC (GE Healthcare, Madison, Wis). ECG, Electrocardiogram.
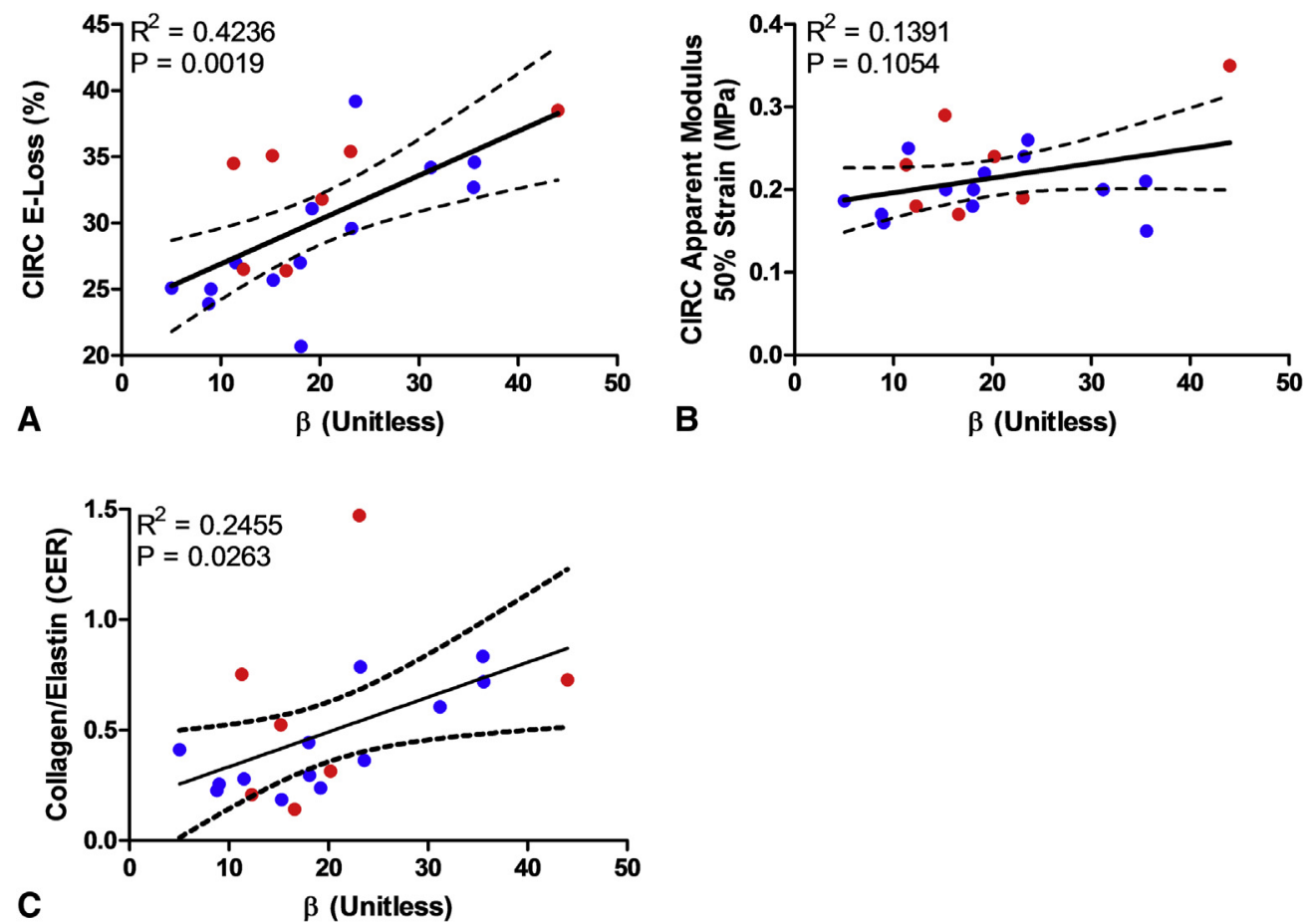

FIGURE E2. Comparison of $\beta$ stiffness index with ex vivo biomechanics and histopathology. A, Ex vivo circumferential energy loss versus $\beta$ stiffness index. B, Ex vivo circumferential stiffness versus $\beta$ stiffness index. $\mathrm{C}$, Aortic wall collagen/elastin versus $\beta$ stiffness index. Data points are distinguished by aortic valve phenotype: tricuspid aortic valve (red dot) and bicuspid aortic valve (blue dot). Circ, Circumferential. 